\title{
VI. Entwicklungszusammenarbeit und Humanitäre Hilfe
}

\section{(2) OpenEdition \\ Journals}

Electronic version

URL: http://journals.openedition.org/sjep/1272

DOI: $10.4000 /$ sjep. 1272

ISSN: 1663-9677

Publisher

Institut de hautes études internationales et du développement

\section{Printed version}

Date of publication: 1 février 1995

Number of pages: 126-144

ISSN: $1660-5926$

\section{Electronic reference}

«VI. Entwicklungszusammenarbeit und Humanitäre Hilfe », Schweizerisches Jahrbuch für Entwicklungspolitik [Online], 14 | 1995, Online erschienen am: 05 Mai 2013, abgerufen am 08 September 2020. URL : http://journals.openedition.org/sjep/1272 ; DOI : https://doi.org/10.4000/sjep. 1272 


\section{Entwicklungszusammenarbeit und Humanitäre Hilfe (1)}

\section{Übersicht}

Die öffentliche und die private Entwicklungszusammenarbeit (EZA) der Schweiz sah sich in der Berichtsperiode einer Vielzahl neuer Aufgaben in den Entwicklungs- und den Transitionsländern bei gleichzeitig zunehmendem Spardruck im Inland konfrontiert. Nebst den traditionellen Instrumenten - technische Zusammenarbeit, Finanzhilfe, humanitäre Hilfe, Massnahmen der Wirtschaftsund Handelspolitik - stellt der politische und gesellschaftliche Wandel in vielen Ländern, begleitet vom weltwirtschaftlichen Strukturwandel, neue Ansprüche an die Entwicklungszusammenarbeit. Die offensichtlich gewordene Interdependenz der Beziehungen zwischen armen und reichen Ländern sowie die Erkenntnis, dass nur kohärentes, langfristig ausgerichtetes Handeln zu nachhaltiger Entwicklung für alle führt, fordert von den Akteuren der EZA nebst der Weiterführung der klassischen Projektarbeit neue Ansätze in der Entwicklungspolitik (Förderung des Demokratisierungsprozesses, des Friedens, Aufbau von Institutionen, Umwelterhaltung u.a.m.). Gleichzeitig erhöhte sich angesichts des zunehmenden Haushaltdefizits des Bundes der Spardruck massiv. Der neue Rahmenkredit für technische Zusammenarbeit und Finanzhilfe beispielsweise wurde durch das Parlament gekürzt. Die jährlich vom Parlament zu billigenden Zahlungskredite für die EZA wurden ebenfalls reduziert. Auch die privaten Hilfswerke verzeichneten 1993 gegenüber dem Vorjahr einen Rückgang der Spendeneinnahmen. Diese Entwicklung zwingt die privaten und die staatlichen Träger der EZA dazu, ihre Kräfte zu bündeln und ihre Arbeit zu konzentrieren. Dies bedeutet konkret, Prioritäten zu setzen: Die bilaterale Unterstützung auf weniger Partnerländer oder Projekte zu verteilen sowie die multilateralen Aufwendungen zu reduzieren. Diese Anstrengungen Richtung erhöhte Entwicklungseffizienz bei knapper werdenden Mitteln müssen langfristig ausgerichtet werden. Die Direktion für Entwicklungszusammenarbeit und humanitäre Hilfe $(\mathrm{DEH})$ hat die Absicht bekundet, die Zahl von heute 18 Schwerpunktländern im Verlaufe der Jahre weiter zu reduzieren. 
In der hier untersuchten Zeitspanne (1993 bis September 1994) sind vom Bund wichtige Dokumente veröffentlicht worden, welche die Basis für die schweizerische Entwicklungspolitik der neunziger Jahre bilden. Es sind dies der Bericht über die Aussenpolitik der Schweiz in den 90er Jahren und der Bericht des Bundesrates über die Nord-Süd-Beziehungen der Schweiz in den 90er Jahren (Leitbild Nord-Süd), die Botschaft über die Weiterführung der technischen Zusammenarbeit und der Finanzhilfe zugunsten von Entwicklungsländern.

Die an der UN-Konferenz über Umwelt und Entwicklung von Rio 1992 und an der Bevölkerungskonferenz in Kairo 1994 gemachten Erfahrungen und die Vorbereitungen für den Gipfel über die soziale Entwicklung und für die Weltfrauenkonferenz (beide 1995) führen alle zur gleichen gemeinsamen Erkenntnis: Die Entwicklungsprobleme müssen im globalen Zusammenhang gesehen und gelöst werden. So sieht es auch der Bundesrat, wenn er in der Einleitung zum erwähnten aussenpolitischen Bericht (Seite 1) schreibt: „Nicht mehr die Statik der bipolaren Nachkriegsordnung, sondern die Dynamik der „Weltinnenpolitik" bildet heute den Rahmen, innerhalb dessen aussenpolitische Konzeptionen entwickelt und überprüft werden". In diesem Verständnis der interdependenten Welt soll die schweizerische Entwicklungspolitik Teil einer möglichst kohärenten Aussenpolitik sein. Das Leitbild Nord-Süd und der Bericht über die Aussenpolitik der Schweiz in den 90er Jahren befassen sich denn auch hauptsächlich mit der Ausgestaltung einer möglichst kohärenten schweizerischen Politik in ihren Aussenbeziehungen. Dieser Themenbereich ist der Schwerpunkt im zweiten Teil dieses Jahrbuches mit dem Dossier über die Kohärenz der schweizerischen Aussenbeziehungen am Beispiel Schweiz-Indonesien (2).

Im Juni 1994 erfolgte ein Urnengang über die Frage, ob sich die Schweiz am Kontingent der UNO-Blauhelme, welche zur Friedenssicherung eingesetzt werden, beteiligen soll. Das Volksmehr viel knapp positiv aus, während das Ständemehr negativ ausfiel und die Vorlage zu Fall brachte.

\section{1. Öffentliche Entwicklungszusammenarbeit}

\section{Jahresbericht DEH/BAWI}

Der von DEH und BAWI gemeinsam herausgegebene Jahresbericht behandelt 1993 die Förderung des Privatsektors, die Entschuldung und die Bevölkerungsfrage und zeigt Beispiele von bilateralen und multilateralen Massnahmen des Bundes auf diesem Gebiet. Die Schweiz setze sich entschieden für die Förderung des privaten Sektors als wichtiger Motor für die autonome Entwicklung eines Landes ein. Die Rolle der privaten Leistungen sei lange Zeit vernachlässigt worden in den Entwicklungsmodellen: „Früher wiesen die Entwicklungsstrategien dem Staat eine allmächtige Rolle zu. Mangels anderer einflussreicher Strukturen war er nicht nur Führer, sondern gleichzeitig wirtschaftlicher und finanzieller Hauptagent des Landes“ (Jahresbericht 1993, S.6). Die Schweiz 
unterstützt hauptsächlich die Förderung der Klein- und Mittelbetriebe und des Handwerks mit dem Mittel der technischen Zusammenarbeit. Ein neues Schwergewicht bildet die Förderung von Organisationen, Institutionen und Verbänden, welche die Strukturen für privatwirtschaftliche Aktivitäten stärken, wie beispielsweise in Vietnam. Die Schweiz hat mit Vietnam eine Reihe von Abkommen zur wirtschaftlichen und finanziellen Zusammenarbeit unterzeichnet. 1993 beteiligte sich die Schweiz an der Entwicklung und am Wiederaufbau des Landes mit 23,3 Millionen Franken, womit Vietnam hinter Indien $(39,7)$ und Indonesien $(38,4)$ an dritter Stelle unter den asiatischen Entwicklungsländern figuriert. Vietnam wird ab 1995 zum neuen Schwerpunktland der Schweiz. Aus Indonesien wird sich die DEH ab 1996 zurückziehen, weil nach ihrer Analyse die Instrumente des Bundesamtes für Aussenwirtschaft (BAWI) die geeignetere Form der Entwicklungszusammenarbeit darstellen.

Thema der DEH-Jahreskonferenz 1994 war die Umsetzung der Agenda 21 und des Rio-Folgeprozesses in der schweizerischen Entwicklungszusammenarbeit.

\section{Gender balanced development}

Die Vorstellung der DEH in der Frage der gleichberechtigten Entwicklung für Frauen und Männer stützt sich auf das Verständnis von Gender balanced development, dem „zwischen den Geschlechtern ausgewogenen Entwicklungsprozess". Gender balanced development löst damit den Begriff „Frauenförderung“ ab. Im Februar 1994 hat die DEH ihre Leitlinien für die 90er Jahre auf diesem Gebiet unter dem Stichwort „transversale Politik“ veröffentlicht. Ein Überdenken der Vorstellungen soll die Widerstände aufbrechen und die Widersprüche beseitigen, die zu Misserfolgen für die Frauen in der Entwicklung geführt haben. Dazu schlägt die DEH „Aktionslinien“ vor für die Zentrale, das Feld und die Regisseure. In einer ersten Phase (1989-93) war die - damals- Frauenförderung mit 50 Stellenprozenten dotiert. 1994 wurde die Kapazität für die zweite Phase auf 170 Stellenprozente aufgestockt.

\section{Südafrika}

Der Verlauf des weiteren erfolgreichen Gesellschafts- und Entwicklungsprozesses in Südafrika ist wohl für die Zukunft der ganzen Region im südlichen Afrika entscheidend. Die Schweiz lehnte während all den Jahrzehnten der Apartheid in Südafrika Wirtschaftssanktionen ab und nahm nicht am UNO-Embargo teil. Als sich Zeichen eines politischen Wandels in Südafrika zeigten, fing die Schweiz im Rahmen der Entwicklungszusammenarbeit an, private Organisationen in Südafrika zu unterstützen (von 1982 bis 1993 mit rund 50 Millionen Franken). Mit weiteren 80 Millionen Franken will sie während den nächsten vier Jahren zum Wiederaufbau- und Entwicklungsprogramm des Landes beitragen. Diese Absichtserklärung machte Bundesrat Flavio Cotti anlässlich seines Besuches in Südafrika im September 1994. 


\section{Palästina}

Der Friedensprozess im Nahen Osten ermöglicht es nun, die Hilfe an Palästina zu intensivieren. Die DEH hat in Jericho ein Verbindungsbüro eröffnet, das Beziehungen zu verschiedenen lokalen Partnern - den palästinensischen Behörden der autonomen Gebiete und privaten Organisationen - aufbauen soll. Der Aufbau in Palästina wird durch einen Rahmenkredit von 60 Millionen Franken für fünf Jahre finanziert, welcher der Bundesrat im September 1993 bewilligt hat.

\section{Ruanda}

In der Entwicklungszusammenarbeit gibt es auch immer wieder Rückschläge. Drastisch hat sich dies in Ruanda gezeigt, einem Schwerpunktland der schweizerischen Entwicklungszusammenarbeit, als der Bürgerkrieg das Land im April 1994 zerstörte. Auch die Schweiz ist vom Konfliktausbruch im April 1994 überrascht worden und hat ihre Zusammenarbeit suspendiert. Ruanda stand 1993 mit einer Unterstützung von 29,4 Millionen Franken an erster Stelle unter den afrikanischen Partnerländern der bilateralen schweizerischen EZA. 1994 leistete die Schweiz hauptsächlich humanitäre Hilfe; die Aufwendungen der humanitären Hilfe und des Schweizerischen Katastrophenhilfekorps machten 1994 rund 27 Millionen Franken aus. Über die zukünftige Weiterführung des Programms in Ruanda bzw. in der Region wird entschieden werden, wenn sich die Lage normalisiert hat.

\section{Entwicklungshilfekomitee (DAC)}

Die Schweiz ist eines der 22 Mitglieder (21 Industrieländer und die EU-Kommission) des Entwicklungshilfekomitees der OECD (DAC). Das DAC spielt eine wichtige Rolle als Koordinationsorgan der bilateralen Hilfe. Im DAC erarbeiten die verschiedenen Geberländer einheitliche Standards für die Entwicklungspolitik. Wegweisend war das DAC beispielsweise in der Definition der Grundsätze für die "gute Regierungsführung", für die Kohärenz der EZA, die partizipative Entwicklung, die Bedingungen der Finanzhilfe oder der Verwendung von Mischkrediten, der statistischen Erfassung und Ausweisung der EZAAufwendungen. (Vgl. dazu den Beitrag von Thomas Greminger über die EZAStatistik der Schweiz im internationalen Vergleich im zweiten Teil dieses Jahrbuches). Die Koordination der Hilfe muss noch weiter ausgebaut werden. Interessenkonflikte sollen sichtbar gemacht, besprochen und möglichst ausgeräumt werden. Dazu braucht es umfassende Informationen. Jedes Mitgliedland liefert allährlich einen Bericht über seine EZA in Form eines Memorandums, und alle drei Jahre prüfen je zwei Länder die EZA eines Landes. Die Schweiz wurde letztmals 1993 geprüft. Die Hilfe wurde als qualitativ gut, jedoch zu gering im Umfang beurteilt. Die Schweiz wurde von den anderen DAC-Ländern „ermutigt, alle Anstrengungen zu unternehmen, ihr mittelfristiges Hilfsvolumenziel von 
0,40 Prozent des Bruttosozialprodukts noch vor dem Jahr 2000 zu erzielen“. (Siehe Jahrbuch 1994).

Zu verschiedenen EZA-Themen (Finanzhilfe, Statistik, Evaluation, Partizipation der Frauen, Umwelt, partizipative Entwicklung und gute Regierungsfühung) sind spezielle Arbeitsgruppen eingesetzt.

\section{Bilaterale Hilfe/ Multilaterale Hilfe}

In der Bevölkerung und bei den Hilfswerken ist die bilaterale Hilfe breit abgestützt, während die multilaterale Hilfe kritischer beurteilt wird. In den letzten Jahren hat sich der Anteil der bilateralen Hilfe des Bundes bei rund 75 Prozent und jener der multilateralen Hilfe bei 25 Prozent eingependelt. 1993 waren es 77 resp. 23 Prozent. In Zukunft dürfte sich der Anteil der multilateralen Hilfe erhöhen. Die Beitragszahlungen an die Bretton-Woods-Institutionen sind ein Grund für diese Erhöhung. Der andere Grund liege in der „Entwicklung der Bedürfnisse in den Entwicklungsländern und der darauf abgestimmten Instrumente der internationalen EZA“, schreibt der Bundesrat (Botschaft 94.029, S. 62). Der Anteil der multilateralen Hilfe dürfte sich bei rund 32 Prozent stabilisieren, wird vermutet. Diese Tendenz sehen die Hilfswerke ungern. Sie befürchten, dass bei noch knapper werdenden Mitteln für die EZA die Einsparungen eher bei der bilateralen Hilfe als bei den multilateralen Verpflichtungen getätigt werden.

\section{Umfrage zur EZA}

Im Abstand von fünf Jahren geben die DEH und die Arbeitsgemeinschaft der Hilfswerke eine Umfrage in Auftrag, um die Einstellung der Schweizerinnen und Schweizer zur Entwicklungspolitik zu ergründen. Im Vergleich zu 1984 und 1989 herrscht heute vermehrt das Gefühl der Machtlosigkeit vor angesichts der weltweiten Probleme. Das allgemeine Klima für die Belange der Entwicklungszusammenarbeit ist in den Jahren der Rezession, des steigenden Staatsdefizits, der europäischen Integration und dem Abseitsstehen der Schweiz rauher geworden. Es ist geprägt von Desinteresse und Spendenmüdigkeit.

Gleichzeitig ist aber auch die Einsicht grösser geworden, das konkrete persönliche Handeln könne einen Beitrag zur Behebung der globalen Probleme leisten. Motiv für die Entwicklungshilfe ist heute die Erhaltung der Umwelt. Noch vor vier Jahren war es die Solidarität. Ebenfalls wichtige Motive sind die Milderung des Flüchtlingsproblems, die Förderung des Friedens und die Verbesserung der Lebensbedingungen der Menschen im Süden. Die Meinungsumfrage bei 1'257 Personen ergab auf die jeweils konkrete Frage folgende Antworten: 79 \% befürworten ein Verbot von Giftmüllexporten in den Süden, $73 \%$ sind für vermehrte Zuckerimporte aus Entwicklungsländern, $71 \%$ befürworten ein Waffenausfuhrverbot in die Dritte Welt, $69 \%$ sind für eine Beschleunigung der Rechtshilfe, um Fluchtkapital auf Schweizer Banken zurückzuführen. 


\subsection{EZ-Ausgaben 1993 (3)}

Die Aufwendungen des Bundes, der Kantone und der Gemeinden bilden zusammen die öffentliche Entwicklungszusammenarbeit. Diese betrugen 1993 insgesamt 1'222 Millionen Franken, davon wurden 1'197 durch den Bund und 25 Millionen durch die Kantone und Gemeinden finanziert. 1992 waren es 1'385 Millionen Franken. Der Rückgang gegenüber dem Vorjahr erklärt sich durch die ausserordentlichen Aufwendungen für den Beitritt der Schweiz zur Weltbankgruppe von 1992. Die Hilfe der Kantone und Gemeinden ist 1993 ebenfalls zurückgegangen, und zwar von 28 Millionen Franken 1992 auf 25 Millionen Franken. Die Übersicht über die EZA und über die Kapitalflüsse liefert Tabelle 18.

Die öffentliche Entwickiungshilfe der Schweiz machte 1993 gemäss der schweizerischen statistischen Methode 0,34 Prozent des Bruttosozialproduktes aus. Noch immer ist die Schweiz damit von der Vorgabe von 0,40 Prozent BSP-

Tabelle Nr. 18

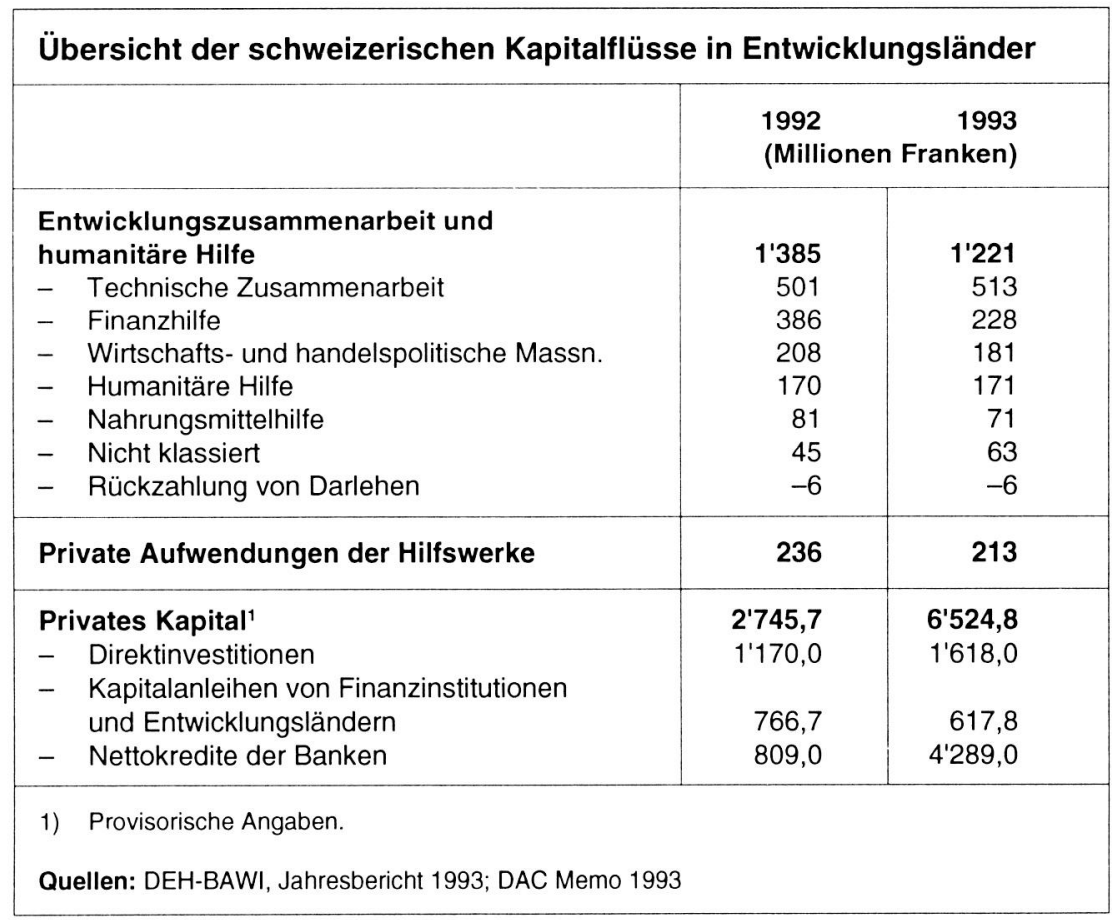


Anteil der Entwicklungshilfe entfernt, die sie aber nach wie vor als Ziel anstrebt. Der Bundesrat hatte in der Legislaturplanung 1991-1995 festgelegt, dass "das Volumen der öffentlichen Entwicklungshilfe bis zur Jahrtausendwende möglichst auf 0,40 Prozent des Bruttosozialproduktes erhöht werden soll“. Gemäss dem Finanzplan für die Jahre 1996-98 soll der Anteil am BSP vorerst bei 0,34 Prozent plafoniert werden.

\section{Aufträge für die Schweizer Wirtschaft aus der EZA}

Nur ein sehr kleiner Teil der schweizerischen Entwicklungshilfe ist gebundene Hilfe, nämlich die Mischkredite und Teile der Nahrungsmittelhilfe, womit die Schweiz im Vergleich zu anderen OECD-Ländern sehr gut dasteht (vgl. den Beitrag von Thomas Greminger über die schweizerische Entwicklungshilfe im internationalen Vergleich). Die wirtschaftlichen Auswirkungen der Entwicklungshilfe sind für die Schweiz sehr interessant. 1992 betrugen die EZA-Aufwendungen 1,3 Milliarden Franken, und es flossen aus der internationalen EZA über 1,5 Milliarden Franken an Schweizer (Expertisen, Beratung) und an schweizerische Unternehmen. (Die Angaben sind detailliert aufgeführt im statistischen Teil). Die Aussage, dass sich Entwicklungshilfe für die Schweiz rentiert, stimmt, muss jedoch dahingehend relativiert werden, dass die Schweiz unabhängig von der konkreten Höhe ihrer Entwicklungshilfe am Wettbewerb um Aufträge aus der - internationalen - Entwicklungszusammenarbeit teilnimmt. Die Wettbewerbsfähigkeit eines Unternehmens ist entscheidend. Somit lohnt sich die EZA generell für die international wettbewerbsfähigen Betriebe der Geberländer.

\subsection{Bilaterale Hilfe}

Der Anteil der bilateralen Hilfe machte 937 Millionen Franken oder 77 Prozent der gesamten EZA-Ausgaben aus. Insgesamt wurden im Rahmen der bilateralen Hilfe 750 Projekte und Programme in 80 Ländern finanziert, wobei sich die umfangreichste Unterstützung auf 18 Schwerpunktländer konzentriert, welche in Tabelle 19 aufgelistet sind. Die Konzentration auf noch weniger Schwerpunktländer soll weiter fortgesetzt werden. Neu hinzukommen wird ab 1995 Vietnam. Drei Länder gelten in Zukunft nicht mehr als Schwerpunktland: Indonesien (siehe Dossier); Kenya, weil die Zusammenarbeit wegen der hohen Korruption der Regierung erschwert ist; die Kap Verden, wo die schweizerische Präsenz abgebaut und auf tieferem Niveau stabilisiert wird, weil sich u.a. Österreich stark in diesem Land engagiert und Koordinationsarbeiten übernehmen kann. Innerhalb der Schwerpunktländer wird langfristig eine Konzentration auf ausgewählte Bereiche erfolgen; die Zusammenarbeit soll sich auf drei bis vier Sektoren beschränken.

Die Instrumente der DEH sind die technische Zusammenarbeit und Finanzhilfe, die humanitäre und die Nahrungsmittelhilfe. Sie machen rund 75 Prozent aller Aufwendungen des Bundes aus. Die Massnahmen des BAWI sind die 
Tabelle Nr. 19

\section{Schwerpunktländer der bilateralen Zusammenarbeit}

Die verstärkte Konzentrationspolitik führt zu folgender Entwicklung:

\begin{tabular}{|c|c|c|}
\hline Lage 1992 & Lage 1994 & Perspektiven 1995-1998 \\
\hline $\begin{array}{l}\text { Westafrika } \\
\text { Benin } \\
\text { Burkina Faso } \\
\text { Kapverden/Guinea Bissau } \\
\text { Ghana } \\
\text { Mali } \\
\text { Niger } \\
\text { Tschad }\end{array}$ & $\begin{array}{l}\text { Benin } \\
\text { Burkina Faso } \\
\text { Kapverden } \\
1993 \\
\text { Mali } \\
\text { Niger } \\
\text { Tschad }\end{array}$ & $\begin{array}{l}\text { Beibehaltung } \\
\text { Beibehaltung } \\
1995 \\
\overline{\text { Beibehaltung }} \\
\text { Beibehaltung } \\
\text { Beibehaltung }\end{array}$ \\
\hline $\begin{array}{l}\text { Ostafrika } \\
\text { Kenia } \\
\text { Madagaskar } \\
\text { Mosambik } \\
\text { Ruanda }^{1} \\
\text { Tansania }\end{array}$ & $\begin{array}{l}1993 \\
\text { Madagaskar } \\
\text { Mosambik } \\
\text { Ruanda } \\
\text { Tansania }\end{array}$ & $\begin{array}{l}\text { - Beibehaltung } \\
\text { Beibehaltung } \\
\text { Beibehaltung } \\
\text { Beibehaltung }\end{array}$ \\
\hline $\begin{array}{l}\text { Asien I } \\
\text { Bangladesh } \\
\text { Indien } \\
\text { Pakistan }\end{array}$ & $\begin{array}{l}\text { Bangladesh } \\
\text { Indien } \\
\text { Pakistan }\end{array}$ & $\begin{array}{l}\text { Beibehaltung } \\
\text { Beibehaltung } \\
\text { Beibehaltung }\end{array}$ \\
\hline $\begin{array}{l}\text { Asien } \text { II }^{2} \\
\text { Indonesien } \\
\text { Nepal } \\
-\end{array}$ & $\begin{array}{l}\text { Indonesien } \\
\text { Nepal } \\
-\end{array}$ & $\begin{array}{l}1997 \\
\text { Beibehaltung } \\
\text { Vietnam statt Indonesien }\end{array}$ \\
\hline $\begin{array}{l}\text { Lateinamerika }{ }^{3} \\
\text { Bolivien } \\
\text { Honduras/Nicaragua }{ }^{4} \\
\text { Peru }\end{array}$ & $\begin{array}{l}\text { Bolivien } \\
\text { Nicaragua/Honduras } \\
\text { Peru }\end{array}$ & $\begin{array}{l}\text { Beibehaltung } \\
\text { Nicaragua/Zentralamerika } \\
\text { Beibehaltung }\end{array}$ \\
\hline \multicolumn{3}{|c|}{ Total der Schwerpunktländer der bilateralen Zusammenarbeit } \\
\hline 20 & 18 & $16+1=17$ \\
\hline
\end{tabular}

1) Die Zusammenarbeit ist nach der Ereignissen vom April 1994 suspendiert. Ob Ruanda später wieder ein Schwerpunktland werden kann, wird neu zu beurteilen sein.

2) Bhutan ist ein Schwerpunktland von Helvetas, das von der DEH unterstützt wird. 1995 wird das Koordinationsbüro in Bangkok geschlossen.

3) Ecuador ist ein Land zweiter Priorität, aber mit Koordinationsbüro.

4) Der Schwerpunkt des zentralamerikanischen Programms wurde von Honduras nach Nicaragua verlegt mit einem regionalen Netzwerk für Zentralamerika.

Vormalige Schwerpunktländer (vor 1990): Tunesien, Paraguay, Kamerun.

Quelle: Botschaft über die Weiterführung der technischen Zusammenarbeit und der Finanzhilfe zugunsten von Entwicklungsländern, Bern, 1994 
wirtschafts- und handelspolitischen Massnahmen. Sie machen rund 15 Prozent der Ausgaben aus. Der Rest verteilt sich auf das Departement des Innern (Bildung und Wissenschaft) und das Finanzdepartement. Anlässlich eines Seminars des Schweizerischen Bundesarchives und der DEH wurde die Forderung aufgestellt, dass sich für eine sinnvolle und kohärente Entwicklungspolitik eine Reform der Verwaltung aufdränge und die Zuständigkeit für die EZA bei einem Departement zusammengefasst werden sollte (4).

Das Bundesgesetz von 1976 schreibt vor, dass die schweizerische Entwicklungszusammenarbeit den ärmsten Ländern und der benachteiligten Bevölkerung zugute kommen soll. Nachdem sich die Mehrzahl der ärmsten Länder in Afrika südlich der Sahara befinden, ist es auch dieser Kontinent, dem am meisten schweizerische Entwicklungshilfe zufliesst (302 Millionen Franken), es folgen Asien (255) und Lateinamerika (87). (Vgl. dazu die detaillierten Angaben im statistischen Teil).

Wir erläutern im folgenden die in der Berichtsperiode veröffentlichte Botschaft über die Weiterführung der technischen Zusammenarbeit und der Finanzhilfe zugunsten von Entwicklungsländern.

\subsection{Neuer Rahmenkredit für technische Zusammenarbeit und Finanzhilfe}

Die Massnahmen der Entwicklungszusammenarbeit werden den vom Parlament für jeweils vier Jahre bewilligten Rahmenkrediten belastet. Die Übersicht über die geltenden Rahmenkredite ist im Jahrbuch 1994 (S. 137) dargestellt. Für die Jahre 1995 bis 1999 hat der Bundesrat einen neuen Rahmenkredit von 3'900 Millionen Franken beantragt, um die technische Zusammenarbeit und Finanzhilfe des Bundes weiterzuführen. Das Parlament bewilligte den Rahmenkredit in der Höhe von 3'800 Millionen Franken. Dieser löst den Ende 1994 aufgebrauchten Rahmenkredit von 3'300 Millionen Franken ab. Die Botschaft sie wurde dem Parlament gleichzeitig mit dem Nord-Süd-Leitbild zugestellt konzentriert ihre Analyse auf die Anstrengungen, die notwendig sind, um eine nachhaltige Entwicklung zu fördern.

Der wirtschaftlichen und politischen Analyse der "Dritten Welt“ geht eine Begriffsklärung voraus. Die Botschaft betont, dass die Kategorisierung der Länder in die "Drei Welten“ nach dem Zerfall der „Zweiten Welt" ihre Bedeutung verloren hat. Die "Dritte Welt" ist heute noch weniger als früher eine politische Handlungseinheit. Vielmehr sind infolge der grossen Kontraste z.B. zwischen den asiatischen Schwellenländern und den afrikanischen Ländern der Subsahara die Interessengegensätze innerhalb des Südens gewachsen. Im Wettbewerb um Kapital und um Aufwertung als Handelspartner zwischen den Transitionsländern des Ostens und den Ländern des Südens droht den ärmsten unter innen eine zusätzliche Marginalisierung. Dem will die schweizerische EZA entgegenwirken. 
Nachhaltige Entwicklung

„Nachhaltige Entwicklung bezeichnet einen Prozess, der von einer vielfältigen, teilweise widersprüchlichen Welt ausgeht und der den Bedürtnissen der gegenwärtigen Generationen Rechnung zu tragen versucht, ohne dabei die Möglichkeit künftiger Generationen zu beeinträchtigen. “... „Nachhaltige Entwicklung bezeichnet ein dynamisches und ausgewogenes Zusammenwirken aller menschlichen und natürlichen Faktoren. Es handelt sich um ein übergeordnetes Ziel, das für alle Gesellschaften gilt. Die Länder des Nordens und des Südens haben zwar für die Umsetzung einer Strategie der dauerhaften Entwicklung unterschiedliche Pflichtenhefte, es geht aber überall darum, sämtliche Bereiche menschlicher Tätigkeit auf das Ziel der Nachhaltigkeit abzustimmen“.... „Die grössten Gefahren, die von einer nicht nachhaltigen Ressourcennutzung ausgehen - Wüstenbildung, Klimaveränderungen, mögliche Erhöhung des Meeresspiegels - würden die Entwicklungsländer unverhältnismässig stärker treffen“... „... eine Globalisierung des westlichen Lebensstandards auf dem Niveau des gegenwärtigen Energie- und Ressourcenverbrauchs ist nicht mehr möglich. Daraus ergibt sich ein schwerwiegendes Dilemma, das der Norden und der Süden gemeinsam angehen müssen. “

(Zitate aus der Botschaft, S. 32-34)

Die technische Zusammenarbeit (1993 513 Millionen Franken) und die Finanzhilfe (228 Millionen Franken) sind vom Umfang her die wichtigsten Instrumente der schweizerischen EZA (60 Prozent der gesamten Ausgaben 1993). Sie werden sowohl bilateral als auch multilateral abgewickelt. Der Rahmenkredit von 3'300 Millionen Franken war Ende 1994 vollumfänglich verpflichtet und wird durch den neuen Rahmenkredit von 3'800 Millionen Franken abgelöst. Der ursprüngliche Wunsch des EDA lautete auf 4'150 Millionen Franken für weitere vier Jahre. Aufgrund der Budgetprobleme des Bundes und der „Stimmung in der Politik" reduzierte der Bundesrat den Antrag für den Rahmenkredit an das Parlament auf 3'900 Millionen Franken, welcher in der Schlussbereinigung im Dezember 1994 in der Höhe von 3'800 Millionen Franken genehmigt wurde.

Rund ein Drittel (1'400 Millionen Franken) soll gemäss Botschaft multilateral verwendet werden, zwei Drittel bilateral (2'500 Millionen Franken). Die Verpflichtungen aus dem neuen Rahmenkredit für die Jahre 1995-1998 sind in Tabelle 20 dargestellt. 
Tabelle Nr. 20

\section{Aufteilung nach bilateralen und multilateralen Trägern der Entwicklungszusammenarbeit: Verpflichtungen 1995-1998}

\begin{tabular}{|l|r|}
\hline & Mio. SFr. \\
\hline I. Bilaterale Programme & $2 ' 500$ \\
a) Asien & 725 \\
b) Afrika & 845 \\
c) Lateinamerika & 300 \\
d) Allgemeine Sektorprogramme & 225 \\
e) Programmbeiträge NGO & 225 \\
f) Andere bilaterale Massnahmen & 180 \\
II. Multilaterale Beiträge & $1 ' 400$ \\
a) UN-Organisationen + andere & 500 \\
b) Regionale Entwicklungsfonds & 375 \\
c) IDA & 525 \\
Total & $3 ' 900$ \\
\hline \multicolumn{2}{|c|}{ Quelle: Botschaft über die Weiterführung der technischen Zusammenarbeit und der Finanzhilfe } \\
zugunsten von Entwicklungsländern, Bern, 1994
\end{tabular}

\subsection{Wirtschafts- und handelspolitische Massnahmen}

Die wichtigsten traditionellen Instrumente in diesem Massnahmenbereich sind die Mischkredite, die Zahlungsbilanzhilfen und die Entschuldungsmassnahmen. Das BAWI will in Zukunft auch neue, den Bedürfnissen der Entwicklungsländer angepasste Finanzierungsinstrumente einsetzen.

\section{Mischkredite}

Die Schweiz nimmt am Exportkreditarrangement der OECD teil. Dieses hat Massnahmen über die Gewährung von Exportkrediten und gebundene Entwicklungshilfekredite beschlossen („Helsinki-Paket“), die 1993 wirksam wurden. Ziel des Arrangements ist die Reduktion der Handelsverzerrungen durch gebundene Hilfskredite und die gezieltere Ausrichtung von Hilfsgeldern auf die Bedürfnisse des Partnerlandes. Die OECD-Länder des Exportkreditarrangements sind sich weitgehend einig, dass für industrielle Projekte $(40 \%$ der bisherigen Mischfinanzierungen) und für Infrastrukturprojekte (insb. Ener- 
gie, Telekommunikation) in städtischen oder gut entwickelten ländlichen Gebieten keine gebundene Hilfskredite mehr gewährt werden sollen. Diese Finanzierung könne der Privatsektor bereitstellen.

Die Umsetzung des OECD-Arrangements schränkt die Vergabe von Mischfinanzierungen durch die Schweiz ein. Dies schreibt das Bundesamt für Aussenwirtschaft, welches für die wirtschafts- und handelspolitischen Massnahmen zuständig ist, im Aussenwirtschaftsbericht 1993. Die Anzahl der Mischfinanzierungs-Aktionen der Schweiz ist deshalb rückläufig, doch werden die Auszahlungen rascher abgewickelt. „Da die im Rahmen von Mischfinanzierungen eingegangenen Verpflichtungen des Bundes rechtsverbindlich sind, war eine erneute interne Umverteilung auf Kosten anderer wirtschaftsund handelspolitischer Massnahmen der EZA unumgänglich... „(Aussenwirtschaftsbericht 1993, S. 94). Die Schweiz unterstützt die Verschärfung der Kriterien für die Anwendung von Mischkrediten und erwartet von den anderen Geberländern, dass alle sich an die neuen Richtlinien halten.

In der Berichtsperiode sind nur wenige Mischkreditabkommen vereinbart worden:

1993 wurde mit Vietnam ein neues Mischkreditabkommen geschlossen mit einem Gesamtbetrag von 25 Millionen Franken (Bundesanteil $50 \%$ ). Gleichzeitig wurde Vietnam auch eine Zahlungsbilanzhilfe von 15 Millionen Franken gewährt, um die Synergie der Hilfe auszuschöpfen. Mit dem Geld soll der institutionelle Aufbau des Bankensektors unterstützt werden.

Thailand wurde 1993 der zweite Mischkredit von 1984 um 13,85 Millionen Franken aufgestockt (Bundesanteil 1/3) zur „Fertigstellung eines Projektes im Energiesektor" (Aussenwirtschaftsbericht). Der Totalbetrag dieses zweiten Mischfinanzierungsabkommens beträgt 78,43 Millionen Franken. Zusammen mit dem ersten Mischkredit von 1979 sind es insgesamt 115 Millionen Franken. In der Presse wird das „Projekt im Energiesektor“ als ökologisch unverantwortbare "Dreckschleuder" bezeichnet (Tages-Anzeiger, 2.3.1994). Es handelt sich um das Kohlekraftwerk von Mae Moh, das Braunkohle von minderer Qualität mit sehr hohem Schwefelgehalt (das sog. Lignit) verbrenne und hohe Mengen von Schadstoffen freisetze. Es mussten nachträglich Schwefelfilter eingebaut werden.

Pakistan wurde eine Aufstockung des Mischkredites von 1987 in Höhe von 20,6 Millionen Franken gewährt (Bundesanteil $40 \%$ ). Der Mischkredit von insgesamt 110,6 Millionen Franken (inkl. Aufstockung) dient zur Finanzierung eines Fernmeldeprojektes im ländlichen Raum.

\section{Zahlungsbilanzhilfe}

Die Zahlungsbilanzhilfe werde auch in Zukunft ihre Bedeutung behalten, ja noch ausgebaut werden, da in einer zunehmenden Anzahl von Ländern wirtschaftliche Reformprogramme anstehen, die so lange unterstützt werden, „bis die Restrukturierungsprogramme wirksam werden und sich in zusätzlichen Exporteinnahmen niederschlagen" (Aussenwirtschaftsbericht, S. 97). Die 
Zahlungsbilanzhilfen schwanken je nach Finanzierungsbedürfnissen der Empfängerländer. Sie können nicht langfristig geplant werden.

1993 erhielten vier Länder Zahlungsbilanzhilfen:

Äthiopien erhielt von der Schweiz 10 Millionen Franken in Form einer Kofinanzierung mit der IDA zur Unterstützung des Strukturanpassungsprogrammes.

Mit Vietnam schloss die Schweiz einen Vertrag über die wirtschaftliche Zusammenarbeit, welcher eine Zahlungsbilanzhilfe von 15 Millionen Franken, den erwähnten Mischkredit von 25 Millionen Franken, sowie einen Beitrag von 10 Millionen Franken zur Finanzierung der Rückstände Vietnams gegenüber multilateralen Instituten beinhaltet.

Mit der Zahlungsbilanzhilfe von 1 Million Franken an Madagaskar wird die Umstrukturierung des Bankensektors gefördert.

Uganda wurde eine Finanzhilfe von 10 Millionen Franken gewährt für prioritäre Importe und zur Erneuerung von Elektrizitäts-Verteilstationen.

\section{Entschuldungsmassnahmen}

Die Entschuldungsmassnahmen sind ebenfalls ein wichtiges Instrument der wirtschafts- und handelspolitschen Hilfe. Sie sind im Kapitel 2 behandelt.

\section{Neue Finanzierungsinstrumente}

Das BAWI kündigt auch im Aussenbericht 1993 wieder (wie im vorangegangenen) die Schaffung von neuen Finanzierungsinstrumenten an, welche den gewandelten Bedürfnissen in den Entwicklungsländern angepasst werden sollen. Die Ausarbeitung des neuen Instrumentariums erfolge in Zusammenarbeit mit der schweizerischen Privatwirtschaft. Ziel sei die konkrete Unterstützung der Privatinvestitionen und generell des Liberalisierungsprozesses in den Entwicklungsländern. „Die neuen Finanzierungsinstrumente sollen insbesondere denjenigen Entwicklungsländern zugute kommen, in denen der Privatsektor trotz positiver Rahmenbedingungen nur zögernd reagiert." (op.cit. S. 95). Pilotprojekte unter dem neuen Finanzierungsinstrumentarium sind in Vorbereitung. Als Beispiel nennt das BAWI die Unterstützung eines Regionalfonds für Leasingunternehmen in Afrika (EDFUND) sowie eines Fonds zur Förderung von Kleinunternehmen in Lateinamerika (PROFUND). Das neue Instrumentarium soll insbesondere innerhalb des neuen fünften Rahmenkredites (ab 1995) realisiert werden.

\subsection{Humanitäre Hilfe}

Die humanitäre Hilfe des Bundes ist ein wichtiges Instrument der schweizerischen Aussenpolitik und leistet einen Beitrag an die weltweit steigenden humanitären Bedürfnisse. Rund 20 Prozent der gesamten öffentlichen Hilfe des Bun- 
des werden für Massnahmen der humanitären Hilfe - sie beinhaltet auch die Nahrungsmittelhilfe - verwendet. Zuständig für diesen Massnahmenbereich ist die Abteilung Humanitäre Hilfe und Schweizerisches Katastrophenhilfekorps (SKH) der DEH. Sie wird geleitet vom Delegierten für Humanitäre Hilfe.

1994 hat DEH-Direktor Walter Fust „Die Strategien der humanitären Hilfe des Bundes für die zweite Hälfte der neunziger Jahre" genehmigt. Sie setzen Akzente für die Tätigkeit vor Ort zu Gunsten der Betroffenen von Natur- und Zivilisationskatastrophen sowie von bewaffneten Konflikten. Die in dieser Form erstmals präsentierte Strategie der humanitären Hilfe basiert auf dem NordSüd-Leitbild für die neunziger Jahre und formuliert die spezifischen Eigenheiten der humanitären Hilfe. Die humanitäre Hilfe ist der Universalität verpflichtet, d.h. jedes Land und alle Bevölkerungsgruppen kommen als Empfänger in Frage. Massgebendes Kriteriuin ist das Vorhandensein einer Notlage. Die Hilfe erfolgt neutral und unparteilich; sie ist an keine politischen Bedingungen geknüpft. Das Kriterium ist für die Schweiz die Achtung der unantastbaren Würde des Menschen ohne Unterschied von Rasse, Geschlecht, Sprache, Religion, politischer Meinung oder sozialer Zugehörigkeit.

Massnahmen der humanitären Hilfe sind die eigenen Projekte und Aktionen des Schweizerischen Katastrophenhilfekorps sowie die Unterstützung durch den Bereich Humanitäre Zusammenarbeit an Programme schweizerischer Hilfswerke und internationaler Organisationen, inkl. IKRK, welche mit Barbeiträgen und Nahrungsmitteln unterstützt werden. Während eines Jahres sind durchschnittlich rund 80 Angehörige des SKH im Einsatz in den Bereichen Soforthilfe, Überlebenshilfe, Wiederaufbau und in der langfristig angelegten Prävention.

Die Aufwendungen für humanitäre Hilfe machten 1993 den Betrag von insgesamt 237 Millionen Franken aus. Darin sind auch die 55 Millionen Franken Beitrag an das Sitzbudget des IKRK und rund 1 Million Franken Beitrag an das Verwaltungsbudget der OIM (Internationale Organisation über Migration) enthalten, welche auf je eigenen Bundesbeschlüssen beruhen und aus separater Quelle finanziert werden. Der Rahmenkredit für die humanitäre Hilfe wurde somit 1993 mit rund 181 Millionen Franken belastet. Die grösste Unterstützung erhielten Ex-Jugoslawien und Ruanda.

\section{Anmerkungen}

1. Dieses Jahr präsentiert sich das Kapitel Entwicklungszusammenarbeit gegenüber den vergangenen Jahren neu. Wir konzentrieren uns auf die Politik der Entwicklungszusammenarbeit und auf neue Elemente der Entwicklungszusammenarbeit, wie sie in der Berichtsperiode (1993 bis September 1994) auftraten, insbesondere der neue Rahmenkredit für technische Zusammenarbeit und Finanzhilfe. Der Themenschwerpunkt im analytischen Teil behandelt ebenfalls einen Aspekt der Entwicklungszusammenarbeit, nämlich die Kohärenz der schweizerischen Beziehungen mit Indonesien. Dort ist auch das Nord-Süd-Leitbild erläutert und wiedergegeben. Ein weiterer Beitrag (von Thomas Greminger) analysiert die statistische Ausweisung der schwei- 
zerischen Entwicklungszusammenarbeit im internationalen Vergleich. Im statistischen Teil (3. Öffentliche Entwicklungshilfe) sind die Aufwendungen der Entwicklungshilfe nach Herkunft, Verwendungsart, Regionen und Sektoren im Detail ausgewiesen und kommentiert. Um Wiederholungen zu vermeiden, sind in der Jahresübersicht die Tabellen des statistischen Teils nicht mehr wiedergegeben.

2. Im Dossier erläutern die Autorinnen und Autoren aus den verschiedenen Aktionsbereichen Verwaltung, Privatwirtschaft, Hilfswerke, Gewerkschaften und Universitäten die Probleme, Mechanismen und Instrumente einer möglichst guten Kohärenz der Entwicklungszusammenarbeit zwischen den beiden Ländern Schweiz und Indonesien.

3. Die Zahlen sind dem Jahresbericht 1993 von DEH und BAWI entnommen. Für die Ländergruppierungen verwenden $\mathrm{DEH}$ und BAWI die Definitionen gemäss OECD. Die OECD definiert Albanien, die Türkei, die Staaten Ex-Jugoslawiens sowie Armenien, Georgien, Aserbeidschan, Kirgistan, Usbekistan, Turkmenistan, Kasachstan, Tadschikistan als Entwicklungsländer.

4. Das Seminar stützte sich auf die umfangreichen Studien über die schweizerische Entwicklungspolitik, welche im Band 19 der Studien und Quellen des Schweizerischen Bundesarchives veröffentlicht wurden: Von der Entwicklungshilfe zur Entwicklungspolitik. Siehe dazu den Beitrag von Beatrix Mesmer und Peter Hug im zweiten Teil dieses Jahrbuches.

\section{Quellen}

Bericht über die Aussenpolitik der Schweiz in den 90er Jahren , Bern, November 1993 (93.098)

Bericht des Bundesrates über die Nord-Süd-Beziehungen der Schweiz in den 90er Jahren (Leitbild Nord-Süd), Bern, Februar 1994 (94.

Bericht zur Aussenwirtschaftspolitik 93/1+2, Bern, Januar 1994 (94.007)

Botschaft über die Weiterführung der technischen Zusammenarbeit und der Finzanzhilfe zugunsten von Entwicklungsländern, Bern, April 1994 (94.029)

DEH/BAWI, Jahresbericht 1993, Bern, 1994

OECD, CAD - Rapport 1993 (Efforts et politiques des Membres du Comité d'Aide au Développement, L'aide en transition), Paris, mars 1994

DEH/BAWI, Memorandum der Schweiz an das DAC 1992 und 1993, Bern, 1993 und 1994

Arbeitsgemeinschaft Swissaid/Fastenopfer/Brot für alle/Helvetas/Caritas, Entwicklungspolitische Stellungnahmen 1993

dito, Die Nord-Süd-Beziehungen in der öffentlichen Meinung, Beilage zu aktuell Nr. 4/ 1994

DEH, Pressedienst (1993, 1994)

DEH, Politik einer gleichberechtigten Entwicklung für Männer und Frauen, Bern 1993

DEH, Die Entwicklungszusammenarbeit und humanitäre Hilfe der Schweiz , 1993/94, Bern, 1994 (Annexe zum Jahresbericht 1993)

Peter Hug und Beatrix Mesmer (Hg.),Von der Entwicklungshilfe zur Entwicklungspolitik, Schweizerisches Bundesarchiv, Bern, 1993

$\mathrm{DEH}$, Abteilung Humanitäre Hilfe und Schweizerisches Katastrophenhilfekorps (SKH), Die Strategie der humanitären Hilfe des Bundes für die zweite Hälfte der neunziger Jahre, Bern, Februar 1994

Tages-Anzeiger, 21.9.1993 (60 Millionen Aufbauhilfe für Palästina) 
Tages-Anzeiger, 2.3.1994 (Schweizer Mischkredite für Thailand)

NZZ, 15.9.1994 (80 Millionen Franken für Südafrika)

Tages-Anzeiger, 27.9.1994 (Bestandesaufnahme in Ruanda)

Tages-Anzeiger, 15,9.1994 (Budget 1995)

\section{Private Entwicklungszusammenarbeit}

\section{Rückgang der verfügbaren Mittel 1993}

Die privaten Aufwendungen für die Entwicklungsländer aus Spendeneinnahmen machten 1993213 Millionen Franken aus; 1992 waren es 236 Millionen Franken. Hinzu kommen die Leistungen des Bundes im Rahmen seiner EZA in Form von Beiträgen an die Programme der Hilfswerke oder in Form von Regieaufträgen (1993: 134 Millionen Franken; 1992: 132 Millionen Franken). Dass die Leistungen des Bundes an die Hilfswerke 1993 stagnierten, ist neu, verzeichneten diese in den Vorjahren doch ein regelmässiges Wachstum. Die Aufwendungen der Kantone und Gemeinden für Entwicklungshilfe waren 1993 wie die privaten Spenden ebenfalls rückläufig (1993: 25 Millionen Franken, 1992: 28 Millionen Franken). Die EZA-Aufwendungen der Kantone und Gemeinden werden nur zum kleinsten Teil (ca. 2 Millionen Franken) von diesen selber durchgeführt, den grössten Teil verwalten die Hilfswerke. Insgesamt verzeichnete die privat verwaltete und durchgeführte Entwicklungshilfe somit einen Rückgang der verfügbaren Mittel von 396 Millionen Franken im Jahr 1992 auf 372 Millionen Franken 1993. Die Hilfswerke sind unterschiedlich von diesem Rückgang betroffen. Insgesamt arbeiten in der Schweiz mehr als 400 Hilfswerke auf dem Gebiet der Entwicklungszusammenarbeit. Die Hilfe der 25 grösseren ist im statistischen Teil ausgewiesen. (1)

Auch für die Osthilfe hatten die Hilfswerke 1993 weniger Mittel zur Verfügung als in den Vorjahren, nämlich noch 15 Millionen Franken gegenüber 19 Millionen Franken 1992 und 21 Millionen Franken 1991. Bei Beginn der Hilfe an die Staaten Osteuropas und der ehemaligen Sowjetunion stand die humanitäre Hilfe im Vordergrund. Diese soll nun allmählich durch technische Hilfe ersetzt werden. Die Hilfswerke Caritas, Helvetas, Intercooperation und Swisscontact haben zum Zwecke der Koordination ihrer Osthilfe 1993 eine Arbeitsgruppe zu Zentralasien gebildet, um die Ausdehnung ihrer Hilfe auf dieses Gebiet vorzubereiten. Dabei könnte Kirgistan ein Schwerpunktland ihrer koordinierten Hilfe werden. Tadschikistan erhält keine Osthilfe des Bundes, weil die Menschenrechte im Land nicht respektiert werden und die wirtschaftlichen und politischen Reformen noch ausstehen. Das Hilfswerk Caritas bereitet in Tadschikistan ein Pilotprojekt für humanitäre Hilfe im Bürgerkriegsgebiet vor und will so der notleidenden Bevölkerung helfen. (2)

Der Rückgang der verfügbaren Mittel dürfte auch für die privaten Hilfswerke - analog wie beim Bund - den Druck zu mehr Konzentration und zu besse- 
rer Koordination der Hilfe erhöhen. „Der Kampf um die Quantität der Entwicklungszusammenarbeit ging 1993 voll weiter“, schreibt die Arbeitsgemeinschaft der Hilfswerke in ihrer Einleitung zu den entwicklungspolitischen Stellungnahmen 1993. Sie fordert den Bund auf, zusätzliche Massnahmenbereiche in der EZA auch mit zusätzlichen Mitteln zu finanzieren. Die nötigen Mehreinnahmen solle sich der Bund durch die Mehrwertsteuer und durch Treibstoffzollerhöhungen beschaffen.

\section{Entwicklungspolitischer Dialog}

Für den Bund sind die Hilfswerke wichtige Partner in der Durchführung der EZA. Der Bund unterstützt die Hilfswerke mit Subventionen an ihre Programme und beauftragt sie mit der Regie eines Teils seiner Projekte. Sie sind andererseits auch wichtige Gesprächspartner im Dialog über die schweizerische Entwicklungspolitik, eine Rolle, die in den letzten Jahren aufgewertet wurde. Federführend im entwicklungspolitischen Diskurs ist dabei die Arbeitsgemeinschaft der fünf Hilfswerke Swissaid, Fastenopfer, Brot für alle, Helvetas und Caritas. Sie hat in der Berichtsperiode in vielen Bereichen Stellung bezogen. Darunter namentlich zu den Themen GATT, Umwelt, Entschuldung, IWF/ Weltbank, Internationale Konferenzen (Bevölkerung) (siehe entsprechende Kapitel).

In ihrer Stellungnahme zum aussenpolitischen Bericht des Bundesrates kritisieren die Hilfswerke die Dominanz der Europafrage und die entsprechende Marginalisierung ganzer Regionen wie etwa Afrika. Die Arbeitsgemeinschaft forderte den Bundesrat dazu auf, die Beziehungen zu Afrika zu einer aussenpolitischen Priorität zu machen. Die Hilfswerke unterstützen den Bund in der Frage des UNO-Beitritts, den sie so rasch als möglich vollziehen möchten. Im aussenpolitischen Bericht fehle jedoch ein Zeitplan für einen UNO-Beitritt, bemängelt die Arbeitsgemeinschaft.

Um den Exekutivdirektoren der Schweiz bei IWF und Weltbank, Daniel Kaeser und Daniel Gerber, einen direkten Einblick in alle Ebenen des Strukturanpassungsprozesses zu geben, organisierte die Arbeitsgemeinschaft eine Reise durch Ghana, an der auch andere Vertreter aus der Verwaltung teilnahmen. Das Ziel war, einen gemeinsamen Lernprozess aus der Strukturanpassungspolitik in Gang zu setzen. Der politische Dialog der privaten Hilfswerke mit der Verwaltung und dem Parlament ist institutionalisiert durch den Einsitz von Vertreterinnen und Vertretern der Arbeitsgemeinschaft in verschiedenen Kommissionen des Bundes. Die Vernetzung der entwicklungspolitischen Arbeit der AG der Hilfswerke mit anderen Nichtregierungsorganisationen erfolgt auch auf europäischem Niveau (EUROSTEP) sowie in thematischen Arbeitsgruppen (Weltbank, Handel, Umwelt usw.). 1993 wurde im Rahmen von EUROSTEP erstmals eine weltweite, d.h. alle Geberländer umfassende Einschätzung der öffentlichen EZA vorgenommen und unter dem Titel „The Reality of AID“ publiziert (3). 
Das Nord-Süd-Leitbild des Bundesrates wurde von den privaten Hilfswerken begrüsst. Das Leitbild erkenne sehr richtig die Probleme und die Zusammenhänge für eine kohärente EZA, es fehle jedoch gänzlich die Erwähnung des dazu erforderlichen Instrumentariums. Zeitgleich mit der Veröffentlichung des Nord-Süd-Leitbildes meldeten die Hilfswerke im April 1994 ihre Forderungen an den Bund für eine Effizienzsteigerung in der Entwicklungshilfe: Der Bund solle sich in Zukunft auf die Koordination der EZA innerhalb seiner Verwaltung und in internationalen Gremien konzentrieren und die operationelle Projektarbeit vermehrt an die Hilfswerke übertragen.

Die Arbeitsgemeinschaft der Hilfswerke erfüllt eine wichtige Koordinationsaufgabe in der Meinungsbildung und in der Informationsarbeit, ohne jedoch den Anspruch zu erheben, sie decke damit die Meinungsvielfalt innerhalb der über 400 privaten Organisationen ab, von denen jede inre besondere Motivation, ihr spezielles Sachgebiet und ihre eigene Mitgliederbasis hat (4).

Kleine Hilfswerke

Von den zahlreichen religiösen und weltlichen Hilfswerken können wir im Jahrbuch jeweils nur ein paar wenige mit inrem Schwerpunkt vorstellen. In den letzen Jahrbüchern sind vorwiegend grössere Hilfswerke vorgestellt worden. Sie sind es auch, welche den Grossteil der Hilfe aufbringen. Die sieben grössten Hilfswerke verwalten 45 Prozent der privaten EZA.

Dieses Jahr stellen wir stellvertretend für viele andere ein kleines Hilfswerk und eine kleine politische Entwicklungsorganisation vor. Kleine Organisationen geniessen in der Bevölkerung oft starke Sympathien, einerseits wegen der guten Überschaubarkeit, andererseits wegen ihrer sachlichen Kompetenz, beschränken sie ihre Hilfe doch oft auf ein bestimmtes Spezialgebiet.

$\mathrm{Zu}$ den kleineren Organisationen zählt die Stiftung Vivamos mejor. Die Stiftung unterhält in sechs lateinamerikanischen Ländern rund 20 Projekte (1993 betrugen die Aufwendungen insgesamt 410'000 Franken). Sie konzentriert sich geographisch auf Lateinamerika und sachlich auf Mutter-Kind-Projekte im sozialen Bereich. Die 1981 gegründete Stiftung sieht in ihrer Arbeit die These „des verlorenen Jahrzehnts“ in Lateinamerika bestätigt. Die Unterstützung von Selbsthilfegruppen sei angesichts des „Niedergangs im Gesundheitsund Erziehungswesen" als Begleiterscheinung der Schuldenkrise und der Strukturreformen um so notwendiger geworden.

Der Solifonds (Solidaritätsfonds für den sozialen Befreiungskampf in der Dritten Welt) ist ebenfalls eine Stiftung, „aus der Einsicht entstanden, dass Entwicklungszusammenarbeit und humanitäre Hilfe an die Dritte Welt nicht genügen, wenn sie nicht von politischer und sozialer Befreiung begleitet sind" (Zitat aus einer Broschüre des Solifonds). Der Solifonds wurde 1983 vom Schweizerischen Gewerkschaftsbund, von der Sozialdemokratischen Partei der Schweiz und dem Schweizerischen Arbeiterhilfswerk (SAH) gegründet. Der Fonds wird von verschiedenen entwicklungspolitischen Organisationen getragen. Er unterstützt befristete politische Aktionen von Basisorganisationen, Gewerkschaften und Menschenrechtsgruppen in der Dritten Welt. Beispiele der 
Unterstützung des Solifonds sind ein Streik bei Everite (Schweizer Multinationales Unternehmen) in Südafrika, Starthilfe für ein Selbsthilfeprojekt von Slumbewohnerinnen auf den Philippinen, Unterstützung für den Lohnkampf von Zuckerrohrarbeitern in Brasilien, Aufklärungsarbeit über die Rechte der indianischen Bevölkerung in Mexiko. Die Aufwendungen des Solifonds für Projekte in Entwicklungsländern betrugen 1993 74'000 Franken.

\section{Anmerkungen}

1. Die Leistungen von über 200 der privaten Hilfswerke im Rahmen der Entwicklungszusammenarbeit mit den Ländern des Südens und des Osten werden alljährlich detailliert ausgewiesen in der Publikation von I.U.E.D./DEH Schweizerische Hilfe für Entwicklungsländer und Oststaaten.

2. Im September 1993 bereisten Vertreterinnen und Vertreter der vier Hilfswerke während eines Monats Kirgistan und klärten die Möglichkeiten von Projekten für technische Zusammenarbeit ab. Die Eindrücke der Reise sind veröffentlicht in einem Dossier in „mosquito“ vom Dezember 1993.

3. The reality of Aid - An independent Review of International Aid, 1993 und 1994. Der Bericht soll in Zukunft jährlich erscheinen, als kritische Ergänzung des offiziellen OECD/DAC-Jahresberichtes.

4. Für Informationen über die Hilfswerke und ihre Schwerpunkte empfiehlt sich die Lektüre ihrer Jahresberichte. Die grösseren Hilfswerke haben auch eigene Zeitschriften und Bulletins, die regelmässig an die Mitglieder versandt werden.

\section{Quellen}

Arbeitsgemeinschaft Swissaid/Fastenopfer/Brot für alle/Helvetas/Caritas, Entwicklungspolitische Stellungnahmen 1993, Bern 1994

dito, Jahresbericht 1993

Schweizerisches Arbeiterhilfswerk (SAH), Die Zukunft gehört allen - Entwicklungszusammenarbeit heute und morgen, Juli 1993

Solifonds-Broschüre 1994

Tages-Anzeiger, 7.9.1993 (The Reality of Aid)

Tages-Anzeiger, 20.4.1994 (Arbeit vor Ort den Hilfswerken überlassen)

mosquito, Dossier Zentralasien, Dezember 1993 